Lothar Störk

Universität Hamburg

\title{
NOTIZEN ZU ZAGA KRESTOS
}

Zu den eigentümlichsten Gestalten der Geschichte Äthiopiens gehört unzweifelhaft Zaga Krestos (Șägga Krəstos), nach eigenem Bekunden ein Sohn des Kaisers Jakob ( $\mathrm{Ya}^{\mathrm{c}}$ əqob), der nach dem Tod seines Vaters 1607 in der Schlacht gegen Susənyos aus seiner Heimat fliehen mußte. Erster Zufluchtsort war Sennar, die Hauptstadt des Funj-Reiches. Weitere Stationen waren das äthiopische Kloster von Dayr al-Muharraq, Kairo, Jerusalem, Konstantinopel und verschiedene italienische Städte, darunter Neapel und Turin.

Überall wurden ihm hohe Ehren zuteil. So wurde er in Neapel vom Vizekönig beider Sizilien, in Turin vom Herzog von Savoyen empfangen. Höhepunkt seines Italienaufenthaltes war aber die von Rekollekten vermittelte Audienz bei Papst Urban VIII. am 18. Mai, 1633. Zu dieser Zeit schreibt der französische Gelehrte Nicolas-Claude Fabri de Peiresc (1580-1637) unter Berufung auf den aus Marseille stammenden Jean Magi, der in Ägypten eine Handelsniederlassung unterhielt:

Le Sieur Magi avoit veu au Cayre le prince fugitif d'Aethiopie Saga Christos, qui est à présent à Rome, il estoit fort jeune prince accompagné de deux serviteurs Abyssins, et portoit des attestations au Bassa du Cayre de sa qualité, luy demandant secours, mais il le r'envoyà en Constantinople, plusieurs chrétiens le reconnaissoient pour celuy qui se disoit. En l'eglise des Abissins du Cayre et tous les chretiens Cophtes. ${ }^{1}$

Im Jahre 1635 zieht Zaga Krestos weiter nach Frankreich, wo er von Ludwig XIII. empfangen und als Protegé Kardinal Richelieus in dessen bei Paris gelegenen Schloß Rueil lebte, wo er auch 1638 seine Tage beschloß.

Über den Anspruch Zaga Krestos, ein vertriebener Prinz zu sein, herrschten von Anfang an Zweifel, wie bereits das Zitat bei Peiresc erkennen läßt. In der gelehrten Welt treffen wir sowohl negative wie auch positive Stimmen. So lesen wir in Johann Heinrich Zedlers Grossem vollständigen Universal Lexikon von 1749:

Herr Ludolf hält dafür, er sey nur ein Betrüger gewesen. Herr Renaudot aber ist gantz anderer Meinung, und scheinet gar nicht daran zu zweiffeln, daß er ein Printz von Königlichen Geblüte in Aethiopien gewesen sey. ${ }^{2}$

${ }^{1}$ Sidney H. Aufrère, La momie et la tempête. Nicolas-Claude Fabri de Peiresc et la «Curiosité Egyptienne» en Provence au début du XVIIe siècle, Avignon 1990, 106.

${ }^{2}$ Positiv: Ernest A. W. Budge, A History of Ethiopia, Nubia and Abyssinia (according to the Hieroglyphic Inscriptions of Egypt and Nubia, and the Ethiopian Chronicles), London 1928, vol. II, 382 f.; ablehnend: JEAN Doresse, Les anciens mona- 
Aber selbst Hiob Ludolf mit seinen abfälligen Bemerkungen, wie «impudentissimus mendax, impostor vix nomen suum scribere potuit sceleratus iste». ${ }^{3}$ muß einräumen, daß Zaga Krestos «eximia corporis species vultus inter gravitatem \& comitatem mirè compositus ut inter alios Principes agens (Bochartus mihi retulit) dignitate formae suavitate morum illos ceu multò inferiores, longè post se relinqueret, $\&$ admirationem aspectu sui omnibus injiceret». ${ }^{4}$ Auf diese Einschätzung Ludolfs gestützt, heißt es bei Zedler gar: «Er ist einer der schönsten Mannspersonen gewesen, die man jemahls gesehen». Von seinem stattlichen, gefälligen Äußeren und seinem einnehmenden Wesen, sowie seiner außergewöhnlichen Libido wissen auch die folgenden literarischen Texte. In seinen zwischen 1657 und 1699 entstanden «Historiettes», Porträts und Anekdoten bekannter Zeitgenossen, berichtet Gédeon Tallemant des Réaux (1619-1692) über Mme Saulnier:5

La Saulnier estant dans la devotion à ce qu'elle disoit, quand le roy d'Ethiopie vint à Paris, elle l'alla voir par curiosité comme les autres; et, sçachant la reputation qu'il avoit pour les choses de nuict, et que comme un géant de l'Amadis, il se servoit dans ses combats d'une antenne au lieu d'une lance, elle eut bientost conclû avec luy. Le mary ne s'en doutoit point; mais des Roches*, $(*$ Michel le Masle, prieur des Roches, portefeuille du Cardinal. Il a de bons benefices.) chanoine de Nostre-Dame, enragé de ce

stères coptes de Moyenne-Égypte (du Gebel-et-Teir à Kôm-Ishgaou) d'après l'archéologie et l'hagiographie, Paris 1971 = Neges Ebrix 3, Lausanne 2000, 219; OSBERT G. S. Crawford (ed.), Ethiopian Itineraries ca. 1400-1524, Cambridge 1958 (The Hakluyt Society, Series II. Vol. CIX, 20); Peter MARTin, Schwarze Teufel, edle Mohren. Afrikaner in Bewußtsein und Geschichte der Deutschen, Hamburg 1993, 94. Folgende Überlegungen machen es wahrscheinlich, daß Zaga Krestos vornehmer Abkunft war: Als Susənyos nach mehreren Jahren des Kampfes, wobei er den (mutmaßlichen) Vater von Ṣägga Krəstos getötet hatte, an die Macht kam, legte die Mutter von Zaga Krestos diesem nahe, Äthiopien zu verlassen (Budge, A History of Ethiopia, 382 f.). Die Tatsache, dass er sich dem Katholizismus zuwandte und zum Glaubensgenossen des Kaisers Susənyos wurde, konnte ihm nicht helfen. Nach der Abdankung von Susənyos richtete die katholische Kirche ihre Aufmerksamkeit auf den Exilierten. Dabei hat es den Anschein als verfolgte die Kurie nunmehr einen einen neuen Plan, bei dem die französischen Kapuziner (anstelle der spanisch-portugiesischen Jesuiten) und ein neuer Kaiser (Zaga Krestos?) als Hauptparteien agieren sollten. Aus diesem Grunde gelangte Zaga Krestos letztendlich nach Paris unter die Obhut Richelieus. Die politischen Entwicklungen in Europa sowie der Tod des äthiopischen Thronprätendenten ließen diese Pläne jedoch scheitern (Korrespondenz mit S. B. Chernetsow in Mai 2003, dem ich auch für bibliographische Hinweise danke).

${ }^{3}$ Hioв Ludolf, Historia Aethiopica, Frankfurt a. M. 1681, Lib. II, cap.VII, 53, 56; Ders., Ad suam Historiam Aethiopicam antehac editam Commentarius, Frankfurt a.M. 1691, $243 \mathrm{f}$.

${ }^{4}$ Ludolf, Historia, loc. cit. 56.

${ }^{5}$ Die Asterisken beinhalten Zusatztexte von Tallemant. 
que Zaga-Christ (il s'appelloit ainsy**) (**Mme de Rambouillet alla voir dans Ramusio, et trouva que les esclaves en Ethiopie estoient marquez audessus du sourcil. On dit qu'on luy trouva cette marque. - Il y a une relation imprimée de son voyage et de sa fuitte, ou plutost un roman; car ce n'estoit en effect qu'un fable.) luy enlevoit ses amours, car on a tout sceû en suitte par une lettre, le fit avertir de tout. Ce des Roches faisoit l'amy de Saulnier, et luy avoit fait vendre sa charge, luy promettant de le faire conseiller d'estat; il ne le put, et l'autre prit des lettres de véteran, car il avoit vingt ans de service. Le mary fait informer des deportemens de sa femme: les amans, voyant cette persecution, resolurent de s'enfuyr, et prirent ce qu'ils purent; mais ils furent arrestez à Saint-Denis. Elle fut mise en religion, où elle traitta avec son mary. Elle disoit qu'elle aimoit mieux quatre mille escus dans son buffet qu'un sot sur son chevet. Zaga-Christ ne voulut point respondre devant Laffemas au Four-l'Evesque, et dit que les Roys ne respondoient qu'à Dieu seul. Pour faire le conte bon, on disoit que Laffemas avoit dit: «Qu'on m' apporte donc ma robe de Jupiter*** (***On l'accusoit d'avoir esté comedien.)». Le feu evesque d'Angers trouvoit ce conte si plaisant, qu'il appelloit sa plus [belle] robe de chambre, Ma robe de Jupiter; et dans son testament, il y avoit un endroit en ces termes: «Item, je legue ma robe de Jupiter». ${ }^{6}$

Die Episode mit dem Gewand Jupiters muß in Paris sprichwörtlich geworden sein, wird sie doch auch von Ludolf angeführt. ${ }^{7}$ Tallemants Notizen belegen, welche Attraktion der exotische Mann in der französischen Hauptstadt war, zu welchen ehelichen Stürmen sie aber auch Anlaß gegeben hat. Übrigens wurde Zaga Krestos schon bald wieder freigelassen und weiter von den Damen bestürmt und mit kostbarem Geschmeide überschüttet.

Tallemants Erwähnung der Lektüre Mme Rambouillets des 1650 erschienenen Reiseberichts des italienischen Humanisten Giovanni Battista Ramusio läßt einmal mehr den Zweifel an der hohen Geburt des Äthiopiers erkennen. In diesem Zusammenhang erwähnt Tallemant einen von ihm als Roman und Fabel abqualifizierten Bericht über die Reise und Flucht des Zaga Krestos. Es handelt sich hierbei um folgendes, $1635 \mathrm{zu}$ Paris erschienene Traktat: SIEUR DE RECHAC, Les estranges evenemens du voyage de son Altesse, le Serenissime Prince Zaga-Christ d'Ethiopie, du grand Empire des Abyssins, was Ludolf als «libellus supra modum absurdus \& fabulosus» bezeichnet. ${ }^{8}$ Aber auch in anderen zeitgenössischen Werken finden sich Ans-

${ }^{6}$ Tallemant des Réaux, Historiettes II, éd. Antoine Adam, Paris 1961 (Bibliothèque de la Pléiade, 151), 257.

${ }^{7}$ Ludolf, Commentarius, 244.

${ }^{8}$ Ludolf, Historia, Lib. II, cap.VII, i). Sieur de Rechac beschreibt in 9 Kapiteln und auf 58 Seiten die Fluchtgeschichte von Zaga Krestos, beginnend mit der Niederlage seines Vaters und endend mit seiner Ankunft in Frankreich. Den meisten Raum nehmen indes die Geschehnisse in Funj-Reich und beim Zug durch die Wüsteneien bis Kairo ein. Im Anhang wird eine Liste der äthiopischen Herrscher gegeben. 
pielungen auf den äthiopischen «Prinzen». So heißt es in den Guy Patin (1601-1672) zugeschriebenen, Borboniana betitelten Memoiren:

Il contrefait assez bien le prince; il est effronté. Quand de belles dames le vont voir, il cajolle et particularise fort avec elles. Il est abondamment pourvu des avantages de la nature; on dit que c'est par là qu'il a gagné les bonnes grâces de Mme S[aulnier]. ${ }^{9}$

Der Dramatiker und Lyriker Tristan l'Hermite (ca. 1601-1655) schreibt ebenfalls über die erotische Begegnung des Äthiopiers mit Mme Saulnier:

C'est touchant une brave femme

Que dame Saulnier on réclame

Qui depuis quinze ou seize jours

Voulut choisir pour ses amours

Le beau prince d'Ethiopie

Qui d'un charbon est la copie...

Der Prinz «dont l'équipage est assez mince» vermochte gleichwohl die Dame zu verführen, die man nunmehr durch die Straßen von Paris eilen sieht:

Pour contempler devant le Cours

Le noir objet de ses amours.

Über die gemeinsame, in einer Arretierung endenden Flucht des Pärchens spottet Tristan:

Que prises avoit de l'office

Dont il n'avoit plus exercise,

Car sa charge vendue avoit

Le bonhomme qui ne sçavoit

Que l'intention de sa femme

Estoit de luy causer diffame,

Et prenant le chemin d'Evreux

Avecque son bel amoureux.

Doncques en bonne intelligence

Et garnis de bonne chevance,

Ils commencoient sans dire adieu

De se déguerpir de ce lieu,

Quand la mine estant esventée

Dame Thisbé fut arrestée

Avec son Pyrame brunet

Que l'on mit dans le Chastelet. ${ }^{10}$

\footnotetext{
${ }^{9}$ Tallemant des Réaux, Historiettes $I I, 1121$.

10 Tallemant des RÉauX, Historiettes II, $1121 \mathrm{f}$.

«Le beau prince d'Ethiopie
}

Qui d'un charbon est la copie...» erinnert an die bei Ludolf, Historia, Lib. II, ch. VII aufgeführte Grabinschrift von Zaga Krestos: 
Claude Malville (1597-1647) schrieb mehrere Gedichte über Zaga Krestos, darunter ein obszönes, an Mme Saulnier gerichtetes, auf den Tod des Äthiopiers. Ein weniger freizügiges, mit den wiederholten Anspielungen auf die Manneskraft («fünfundzwanzig» bzw. «fünfzehn Stöße») doch immer noch deutliches lautet:

Quinze et vingt coups! Je frisonne;

Un si grand succès m'étonne;

Mais du corps et de l'esprit

Ce diable de Zaga-Christ

Fut une estrange personne.

Sa procédure estoit bonne,

De s'excuser à sa Donne

Quand seulement il en fit

Quinze.

Bien que la France foisonne

En gens dont l'ardeur félonne

Fait presque autant qu'elle dit,

Que le plus fort sur le lit

En approche, et je luy donne

$$
\text { Quinze. }{ }^{11}
$$

1759 erschien The History of Rasselas, Prince of Abissinia des englischen Schriftstellers Samuel Johnson (1709-1784). In diesem moralisch-didaktischen Roman fliehen der Kaisersohn Rasselas, seine Schwester Nekayah, deren Dienerin Pekuah und der Philosoph Imlac nach Kairo, um dort die Lebensverhältnisse anderer Stände und die Quellen des Glücks zu studieren. Nun war Johnson die äthiopische Thematik nicht fremd, hatte er doch eine gekürzte, 1735 erschienene Übersetzung des Reiseberichts von Jeronimo Lobo angefertigt, ${ }^{12}$ doch könnte auch das abenteuerliche Leben Zaga Krestos' ihn zu seinem Roman angeregt haben.

\section{«Cy gist le Roy d'Ethiopie}

l'Original ou la copie».

Es scheint allerdings mehr als fraglich ob einem Gast des französischen Staates ein solch verunglimpfendes Epitaph gewidmet wurde, zumal Richelieu den Tod seines Schützlings um vier Jahre überlebt hat! Es dürfte sich doch wohl eher um eine literarische bezw. im Volksmund zirkulierende Zuschreibung handeln.

${ }^{11}$ Tallemant des RÉAux, Historiettes II, 1122 f. Zu dem herkulischen Sexualvermögen vgl. man die in vielen Sprachen üblichen Wendungen, z.B. ERNEST BorNEMANN, Sex im Volksmund. Der obszöne Wortschatz der Deutschen, 2 Bände, Reinbek bei Hamburg 1974.

${ }^{12}$ Unter dem Titel: A voyage to Abyssinia. By Father Jerome Lobo ... With a continuation of the history of Abyssinia down to the beginning of the eighteenth century, and fifteen dissertations on various subjects, relating to the history, antiquities, government, religion, manners, and natural history of Abyssinia, and other countries mention'd by Father Jerome Lobo, By Mr. Le Grand, London: A. Bettesworth, and C. Hitch, 1735. 


\section{SUMMARY}

The article brings to light some European literary evidences concerning «Zaga Krestos» (Ṣägga Krəstos), a $17^{\text {th }}$-cent. Ethiopian nobleman, possibly related to the royal Solomonic line. Having fled from Ethiopia, he eventually came to Europe, enjoing the benevolence of European ruling houses and the head of the Catholic Church. Finally, he settled in Paris, under the auspices of the highest officials of the French monarchy. Even though there could have been some political projects in which Zaga Krestos was supposed to take over an important role, he became widely known mostly due to his impressive appearance and scandalous affaires with the French high society ladies. 